Health \& Medicine | Dr Nikolai Sopko

\section{New therapy offers hope for wound healing in severely burned patients}

Serious burns are difficult to
treat and can cause many
complications. A common
treatment for these injuries
is a split-thickness skin graft,
where a thin layer of healthy
skin is taken from elsewhere on
the patient's body, meshed and
transplanted onto the wound
bed. Unfortunately, this type
of treatment can lead to graft
failure, contracture and scarring.
PolarityTE, Inc., a biotechnology
company in Salt Lake City,
Utah, USA, has developed a
revolutionary new treatment,
SkinTETw, for burns and other
skin defects, including chronic
wounds and traumatic injuries.
SkinTETM has been proven to
facilitate burn reconstruction and
wound healing by regenerating
fully functional new skin.
he human body has a remarkable
ability to heal itself. Sometimes,
though, the damage is just too severe, and even with the best medical care, the prognosis is poor. This has long been the case
serious burns. Third-degree burns - where the burn fully penetrates all three layers of the
skin - are the most difficult to treat. These patients suffer severe pain and are at high risk of infection, leading to treatment with intravenous antibiotics, as well as other clinical complications. If the burn is extensive, the patient may require a skin graft - a therapy that has been used for decades. This process uses a thin layer of healthy skin from elsewher on the patient's body which is meshed and grafted to the damaged area. Unfortunately, this treatment is imperfect and does not always result in successfu wound healing

SPLIT-THICKNESS SKIN GRAFTS A type of skin graft commonly used in skin grafting. This involves transplanting a thin piece of donor tissue, comprising the outermost layers of the skin: the epidermis and part of the dermis.
The graft is usually taken from a part of the body that heals well, such as the thighs or buttocks.

The advantage of a split-thickness skin graft is that the donor site normally the deeper part of the skin is leftintect. However, there are some disadvantages Only the outermost part of the skin is used, so the wound site does not recover its full function. Split-thickness skin does not contain sweat or oil glands, or hair follicles. So, although split-thickness skin grafts can effectively function as a barrier, they may not aesthetically match the surrounding skin, for example in colour or contraction of the donor skin and scarring.

Full-thickness skin grafts are also possible but leave a deeper wound that must be completely closed in order to heal and are limited by a 1.1 expansion ratio. small and are not suitab for coving large wounds. Both full-thickness and split-thickness skin grafts have a failure rate of around $30 \%$.

\section{A NEW OPTION}

Polarty TE has developed an innovative texture. There are also risks of graft failure, reatment, SkinTE ${ }^{T M}$, for burns and oth wounds and traumatic iniures Polarity is a biotechnology company that is focused on discovering, developing and designing regenerative tissue products. SkinTE' is a novel, firstof-its kind treatment. The advanced technique has the potential to eliminate the unfavourable results of traditional treatments, like split-thickness skin grafts.

SkinTE', is an autologous homologous skin construct (AHSC). "Autologous" because the tissue comes from the patient's own body, and "homologous" because the donor graft will perform it is applied.

The company has established a seamless rocess for deploying SkinTE'". First, the healthcare provider removes arity $T$. piece of healthy, full-thickness skin from the patient. Only a small sample of skin is needed to cover a large wound area leading to minimal harvest-site morbidity This differs from other methods which have been developed with a similar aim but use artificially manipulated homogenous cells. The skin harvest is packed in a sterile, temperaturecontrolled container. The sample is returned to Polanty TE's laboratory and the tissue is processed into AHSC or Skin TE Once processed, SknTE is returned within $48-72$ hours to the healthcare . SkinTE'm, contained within a syringe, is applied over the wound The area is then dressed and treated akin to other types of skin grafts. Over time, SkinTE'm grows and expands to become full-thickness skin with a histology comparable to native skin. Throughout the process, healthcare professionals can communicate with a PolarityTE team of Clinical Science Managers. Treatment with SkinTE" has been proven to regenerate skin wit all layers, appendages and has even produced function such as sensation, sweat and pliability

The development of SkinTE ${ }^{m}$ was inspired by recent research that hows that skin is more than just

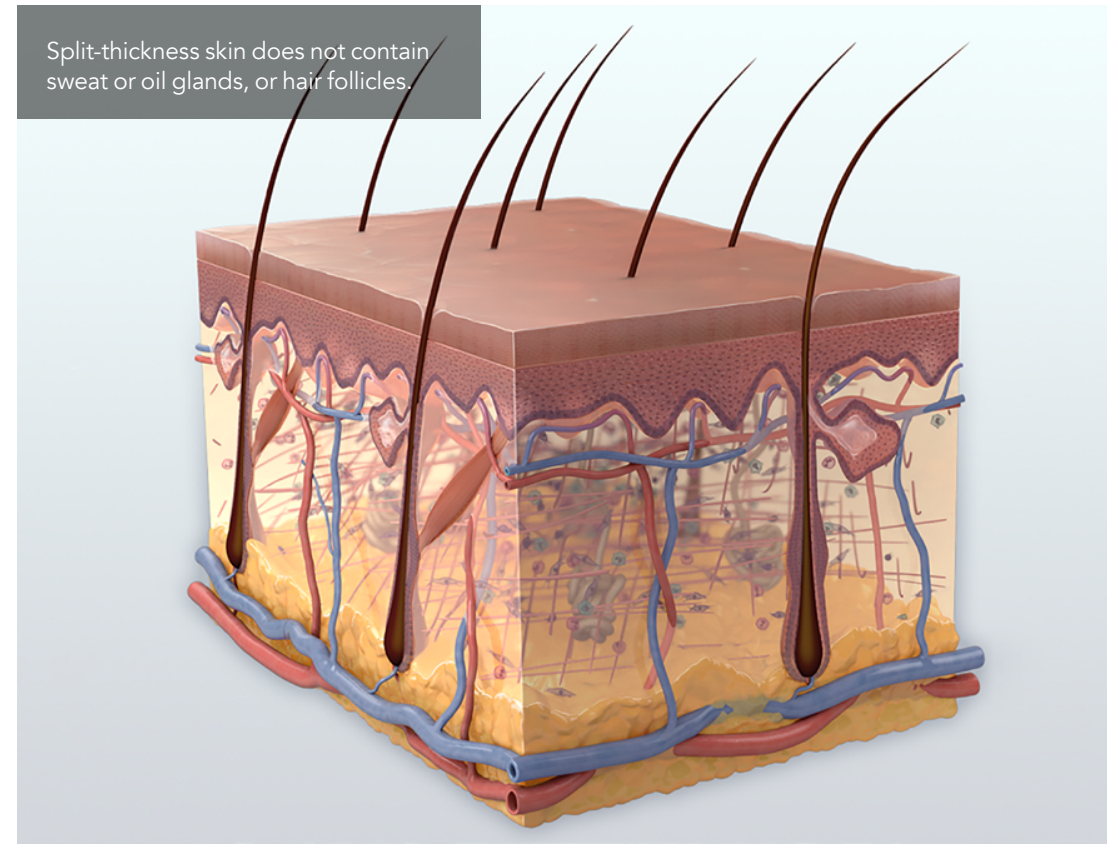

Skin is a complex biological organ, with roles in cell signalling, metabolism and protein synthesis.

organ, playing major roles in cell signalling, metabolism and protein synthesis. It is also a crucial part of the nervous system, immune defence mechanisms and hormonal functions. This shows the importance of utilising full-thickness skin, with a complete heal wounds with a wide range of size and severity

As well as burns, SkinTE'" can be used to ic wounds, including diabetic foot and venous stasis ulcerations, acute

traumatic wounds and amputation sites. teould also serve as a viable replacement of failed skin gratts, to replace scar tissue, PROMISING RESULTS SkinTE'm has already been used in a variety of clinical settings, ranging from intensive care units to wound care centres and outpatient clinics. Polarity TE is currently ninning milicentred, controll standards of $\mathrm{U}$ Up to 16 centres in US are expected to participate in each

AHSC Workflow for clinic setting

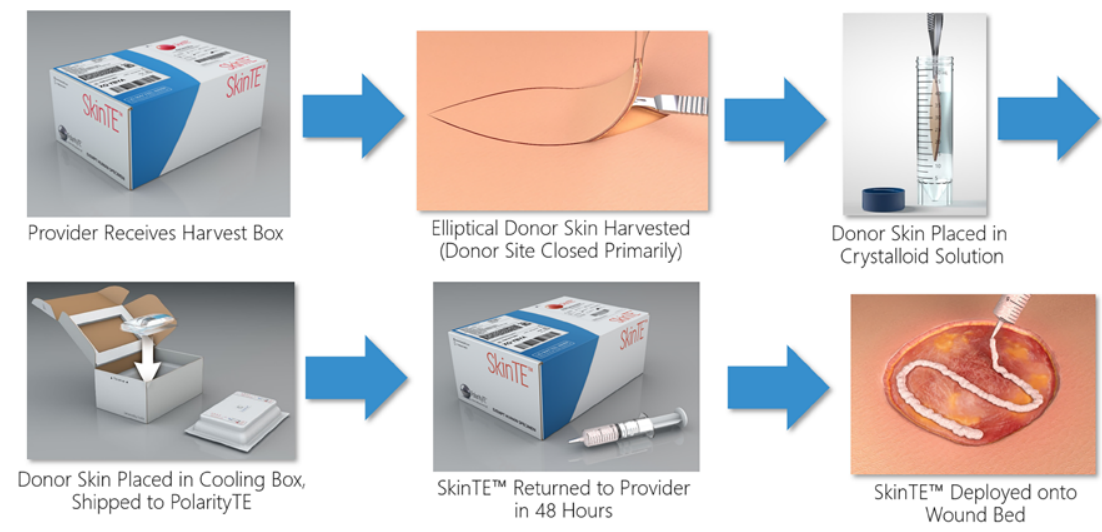

The workflow for clinicians is simple and easy to follow. 


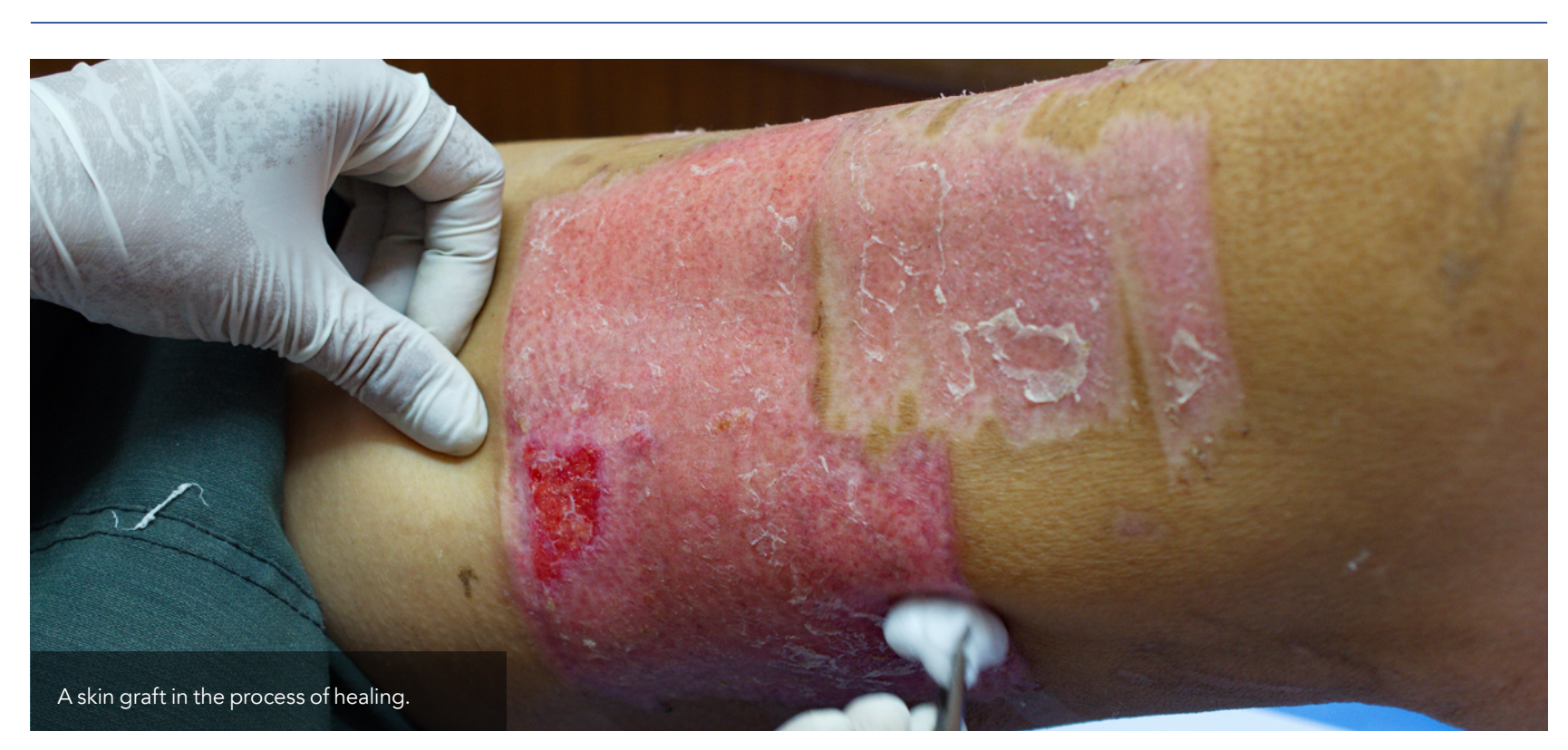

SkinTETM offers the potential for better treatment of extremely serious conditions and injuries.

trial. In addition, several clinical trials are specifically comparing SkinTE ${ }^{\text {tw }}$ to
split-thickness skin grafts in burn patients. Two further trials are evaluating the new treatment when applied to diabetic foot ulcers and venous leg ulcers.

SkinTE" has already produced very encouraging results. In one particular case, SkinTE" was used to treat a patient who had suffered very serious burns in a house fire. The 45-year-old woman had burns to $75 \%$ of her body surface, including on her arms, legs and torso. She developed multiple complications, injury. Due to the extent of the injuries, the patient had little healthy skin that could be used for conventional skin grafting.

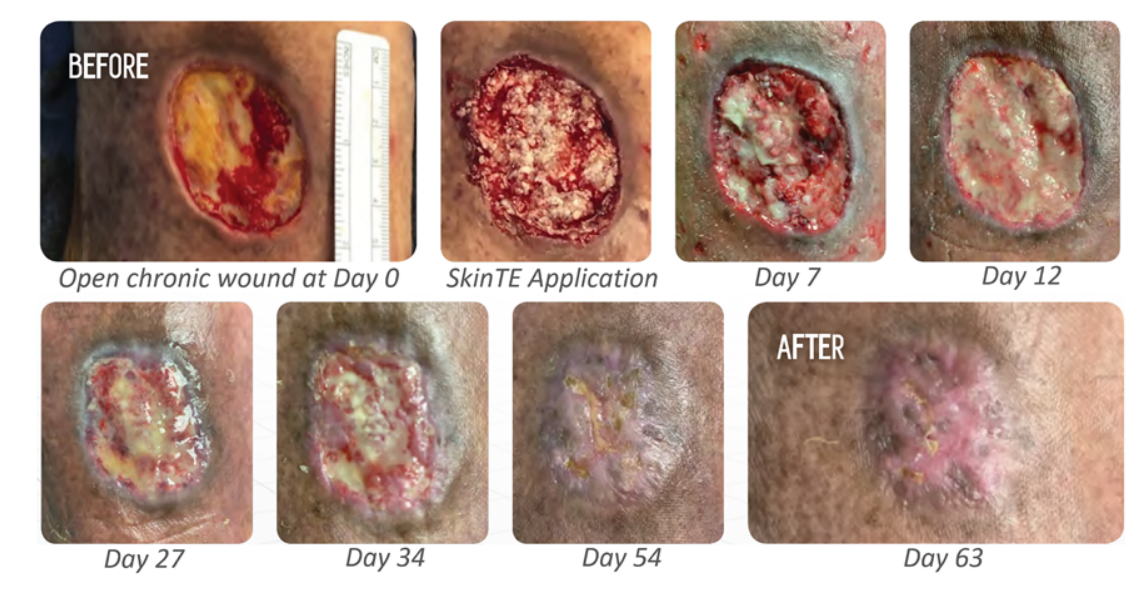

A small sample of healthy skin was take from the patient's abdom PolarityTE ${ }^{\oplus}$ harvest kit. The sample was processed into SkinTE'm at Polarity TE and applied to the patient's wounds two days after the small donor sample was taken. SkinTE" was applied to the time, split-thickness skin grafts were used to treat the burns on her arms, using skin taken from the front of her torso.

By six weeks after SkinTE ${ }^{m}$ was deployed, full epithelialisation (the spread and wound) This is the first step in the formation of new skin, where the new cells act a barrier. By eight weeks, the patient was full function, as well as repigmentaped and normalization. Unfortunately, the patient experienced contraction (when the graft shrinks) and irritation of the skin grafts applied to the wounds on her arms.

In this case, SkinTE ${ }^{\text {tw }}$ was an effective treatment for a patient with extensive, full-thickness burns. SkinTE"'regenerated full-thickness skin, and developed of the affected area and ultimately, improving the patient's quality of life. Like most cases, only a single application defects, in bures and cond titons. Dr Nikous Sopko and his research team believe that SkinTE"' has multiple benefits including time, which can ultimately lead to shorter hospital stays and improved healthcare parts of the body, leading to minimal harvest-site morbidity. The treatment also avoids some of the complications often seen with traditional split-thickness skin grafting. If PolarityTE's clinical trials continue to produce encouraging results, SkinTE could become established as a standard treatment for patients with

serious burns, chronic wounds, and oth
debilitating cutaneous conditions.

Decades-old chronic wound closed with SkinTETM (outcomes may vary).

\section{Behind the Research}

Nikolai Sopko, MD, PhD aster

pigmentation, improving the appearance of SkinTE'"' was needed.

A BRIGHTER OUTLOOK

The novel technology behind SkinTEm the ability to improve the function of new skin and the reduction in healing economics. SkinTE' removes the need for large skin grafts to be taken from other

Research Objectives

PolarityTE has developed products that enable regeneration of full-thickness skin with all layers and appendages, with recent research focusing on chronic wounds.

\section{Detail}

123 N Wright Brothers Drive Salt Lake City

UTA

Bio

Nikolai Sopko, MD, PhD is the Chief Scientific Officer and Medical Director at PolarityTE, Inc where he applies his passion for patient care and basic science research to develop novel regenerative therapeutics for clinically challenging diseases. He obtained his MD with honours from Case Western Reserve University and his $\mathrm{PhD}$ in stem cell biology at the Cleveland Clinic. He completed his surgical training at the Johns Hopkins James Buchanan Brady Urologic Institute as a Physician Scientist Scholar where he focused on regenerative reconstruction, tissue engineering, gene therapy, transplant modulation, and tumour biology. Dr Sopko has over 60 published peer reviewed articles and book chapters and is an award-

Collaborators

- David Armstrong DPM, MD, PhD

- Anand Kumar MD

\section{References}

Granick, M. et al. 2019. In Vivo Expansion and Regeneration of Full-Thickness Functional Skin with an Autologous Homologous Skin Construct: Clinical Proof of Concept for Chronic Wound Healing. Int Wound J. 2019;1-6. https://
onlinelibranywileycom/doiffull/10.1111/iwi.13109 Patterson C, Stark M, Sharma S, Mundinger GS. "Regeneration and Expansion of Autologous Full-Thickness Skin Through a Case Reports. 2019: 00:1-7. Published November 6th, 2019. https://onlinelibrary.wiley.com/doi/10.1002/ccr3.2533

Armstrong, D. et al. 2019. 45-LB: Results of a Pilot Evaluation of a Novel Autologous Homologous Skin Construct Treatment of Diabetes 2019 Jun; 68(Supplement 1). https://diabetes.
diabetesjournals.org/content/68/Supplement_ 1/45-LB Smith S. et al.2019. Coverage of bilateral lower extremities with fully-functional, full-thickness skin following treatment with a novel Autologous Homologous Skin Construct. Abstract 2019.

Mundinger, GS. et al. 2019. Novel therapy for complete . homologous skin construct (Poster). Eplasty. Presented at Symposium for Advanced Wound Care - Spring 2019 (San Antonio, TX). May 10th, 2019. http:///www.eplasty.com/images/ poster/bigposter/SAWC Burn\%20Revision\%20Case\%20

https://wnw.polarityte.com/

\section{Personal Response}

Could the technology behind SkinTETM be used to develop treatments for other types of injury or chron

II The technology behind SkinTETM is incredibly powerful, in part, because it harnesses our body's remarkable ability for repair it has not evolved to deal with difficult-to-treat injuries such as complex traumatic wounds that can occur in motor vehicle accidents or chronic wounds that can occur with ageing and exposure to chronic diseases. The technology helps optimise the body's healing potentia see more and more encouraging clinical results in these settings. - Dr. Nikolai Sopko 